\title{
El rol de la resiliencia socioecológica en la agricultura campesina sostenible.
}

\section{The socioecologic resilence role in sustainable farmer agriculture.}

\author{
Marilyn Gonzalez Urrutia
}

Universidad de la Frontera, Facultad de Ciencias Agrarias y Forestales, Programa de Magíster en Recursos Naturales, Casilla 54-D, Temuco, Chile. Email: mgonzalezurrutia@gmail.com

Distintas líneas temáticas y de investigación se han derivado y escrito a partir del concepto de Resiliencia como propiedad de los metales en mecánica, el que luego fue derivado al ámbito de ecología de sistemas a través de la propuesta de Holling1 (1973) como una manera de ayudar a comprender la dinámica no lineal que se observan en los ecosistemas. Al respecto, una de las definiciones mas connotadas propuesta por Folke et al. (2010) la mencionan como "la capacidad de un sistema para absorber las perturbaciones y reorganizarse, mientras se somete a cambio de manera que aún se conserva esencialmente la misma función y estructura, y por lo tanto, la identidad", es decir, la capacidad de cambiar a fin de mantener la misma identidad2.

Existen muchos trabajos que mencionan las distintas disciplinas que han derivado de este concepto, siendo fin de evaluar en este breve comentario el desarrollo en ecología de sistemas. El planteamiento de su teoría y aplicación ha sido más bien generada dada la inseguridad generada a partir de los informes del cambio climático y sus manifestaciones que indican que este afectará ampliamente la economía. Desde este enfoque la aplicación de este concepto también se ha aplicado naturalmente en granjas de cultivo, puesto que los agricultores siempre han tenido que encontrar soluciones para hacer frente a eventos inesperados como e.g., sequías, inundaciones, enfermedades de los cultivos o animales, con las incertidumbres del mercado o con cambios en la disponibilidad de mano de obra familiar (Darnhofer, 2014: 462). Es por esto que desde el año 2000 se centró una parte de la investigación en la resiliencia socioecológica, la que es vista como una forma importante de prever y adaptarse a los posibles cambios (Malaskaedidi y Karami, 2013:1). Este término se refiere a la capacidad de un sistema que resiste choques, para moderar los daños potenciales de los peligros, todavía teniendo la misma identidad (misma estructura básica y modos de funcionamiento), y, posiblemente, para mejorar y avanzar en el autoaprendizaje y adaptación, para hacer frente a un evento, así como la recuperación durante el post-evento (Malaskaedidi y Karami, 2013:4-5). Al considerar este concepto en los sistemas socio-ecológicos (SES), la definición se amplía para incluir la capacidad del sistema social para organizarse y aumentar su capacidad de adaptación a las condiciones cambiantes en su entorno socioeconómico y natural (Resilience Alliance 2007). Así, el elemento humano añade como aporte al concepto de resiliencia ya que los humanos a través de su capacidad de visualizar, prever y planificar, pueden mejorar la capacidad de recuperación de un sistema. Esto muestra que existe una completa vinculación entre el ambiente ecológico o natural y la administración del ser humano sobre sus recursos.

Un agroecosistema se puede definir como un ecosistema gestionado con la intención de producir, distribuir y consumir los alimentos, combustible, y fibras. Sus límites abarcan el espacio físico dedicado a la producción, así como los recursos, infraestructura, los mercados, las instituciones y las personas (Cabell y Oelofse 2012). El agroecosistema opera simultáneamente a múltiples escalas y

${ }^{1}$ El propone el término como modelo para analizar procesos de auto-organización en situaciones de cambio y frente a perturbaciones, alejadas de dinámicas lineales, siendo una medida de la magnitud de perturbación de un sistema socioecológico (SES) el que puede absorber previamente al cambio de estado que de ello resulta.

2 Para una visión general de la teoría de la resiliencia, incluyendo el ciclo de adaptación y panarquía (Cabell y Oelofse 2012) reúne bibliografías de trabajos previamente citados y no incluidas en esta revisión. 
jerarquías anidadas, desde el campo para el mundo porque ningún agricultor opera en un vacío, y las decisiones que él o ella hacen se basan en gran parte en influencias externas (Darnhofer et al. 2010). Cabell y Oelofse (2010) a través de su investigación respaldan investigaciones anteriores, mencionando que todos los agroecosistemas se mueven a través de las cuatro fases del ciclo de adaptación: el crecimiento/explotación, conservación, liberación, y reorganización / renovación ${ }^{3}$. Un enfoque de resiliencia trata elementos biofísicos, sociales y económicos de una región como componentes de un único sistema social-ecológico (SES), destacando la capacidad de un SES para continuar ofreciendo productos y servicios a las personas, y las ventajas y desventajas asociadas (Walker et al. 2009).

Una granja no puede estudiarse de manera aislada, sino más bien se debe entender las prácticas agrícolas: la granja debe ser entendida como incrustada en un territorio, una localidad, una región, con su entorno, agroecología específica, oportunidades económicas y sus valores culturales (Darnhofer et al. 2012: 4). En las granjas familiares de beneficios reportados a corto plazo, el comportamiento de búsqueda tiende a ser mitigado por un compromiso a largo plazo para la agricultura como forma de vida (Darnhofer, 2010: 213). De este modo la capacidad de recuperación de la agricultura tiene por objeto la finca como un sistema, para preservar sus funciones, no más bien a la preservación de las actividades individuales de producción en la granja (Darnhofer, 2010: 215). Esto significa que debe existir un reacondicionamiento natural de actividades según el comportamiento de la granja en donde según Malaskaedidi y Karami (2013:9) esto sucede porque la resiliencia es una condición previa necesaria para la sostenibilidad de los sistemas agrícolas, siendo un sistema de agricultura resiliente el que puede conservarse por si mismo y con la misma estructura durante mucho tiempo, también siendo sostenible. Así un sistema agrícola tiene componentes que son unidos por la interacción y la interdependencia, funcionando dentro de un determinado límite para alcanzar algunos objetivos agrícolas específicos, teniendo dimensiones sociales, ecológicas, y dimensiones económicas.

En el contexto de la agricultura, una crisis puede ser entendida como un momento cuando algún componente del sistema agrícola campesino familiar se comporte disfuncional, como por ejemplo, un agroecosistema degradado. Como tal, las crisis no necesariamente deben ser entendidos como hechos "negativos" pues pueden abrir oportunidades para revalorar la situación actual, desencadenando movilización social y estimular el aprendizaje a través de experiencias de reconsideración y el conocimiento (Darndofer 2014: 9). Estas crisis pueden entonces ser una 'ventana de oportunidad', que permite transformación y cambio, pudiendo convertirse en ventajosas en algunos casos.

En un sistema agrícola, la granja, la resiliencia depende de la capacidad de integrar y equilibrar las tres capacidades (buffer, adaptación y transformación), permitiendo de este modo hacer frente a crisis repentinas, sorpresivas e impredecibles así como también pudiendo presentarse como cambios de evolución lenta (Darnhofer et al. 2010a). Por otra parte, la capacidad de recuperación es una propiedad emergente de un sistema, que surge de la única interacción entre el agricultor-granja-y su particular contexto, lo cual implica que no hay dos sistemas iguales, pues lo que hace que uno sea resiliente puede no necesariamente funcionar en otra granja. Así los agroecosistemas encarnan toda la complejidad que un SES posiblemente puede tener, por lo que es casi imposible dar cuenta de todos los factores que contribuyen a la resiliencia, tanto ahora como en el futuro (Darnhofer et al. 2010a).

En la capacidad de recuperación destaca la capacidad de las granjas de adaptarse ante el cambio, dependiendo de su capacidad de recuperación del contexto. Una granja está vinculada a las dinámicas territoriales y sectoriales más amplias, lo que necesariamente influye, pero no determina, las opciones disponibles para una granja. De hecho, una granja necesita participar en co-evolución con el contexto ecológico, social, económico y político (Darndofer, 2014:10). Por esto Cabell y Oelofse (2010) indican que es necesaria una reorganización radical de las instituciones políticas, económicas y sociales puesto que los

${ }^{3} \mathrm{Al}$ igual que todos los sistemas socioecológicos (SES) que existen y operan dentro de un entramado jerárquico de otros sistemas naturales y antrópicos, también lo hacen sus ciclos de adaptación, siendo organizado este anidamiento de ciclos de adaptación bajo una jerarquía: la panarquía. 
sistemas agrícolas son demasiado complejos para ser medidos en cualquier forma precisa y, como tal, están mejor servidos por el desarrollo de normas de resiliencia generales que son aplicables en toda escalas de tiempo y espacio.

De manera simple, apoyándose en las preguntas de Malaskaedidi y Karami (2013:2), la resiliencia busca resolver las siguientes preguntas: ¿Cómo los agricultores pueden soportar la escasez de agua y ser sostenibles? ¿Puede la aplicación teórica de la resiliencia aumentar la comprensión de la sostenibilidad? ¿Cuál es el papel de la teoría de la resiliencia en la consecución de una agricultura sostenible dada la escasez de agua como una crisis ambiental? Por lo tanto, para investigar el papel de la resiliencia para alcanzar una agricultura sostenible, es precisa una visión sistemática y considerar la capacidad de recuperación a escalas tanto social como ecológica.

Referencias.

Cabell, J.F. and M. Oelofse, 2012. An indicator framework for assessing agroecosystem resilience". Ecology and Society, 17: 18.

Darnhofer, I., 2010. Strategies of family farms to strengthen their resilience. Environmental Policy and Governance 20: 212-222.

Darnhofer, I., 2014. Resilience and why it matters for farm management". European Review of Agricultural Economics, 41: 461-484.

Darnhofer, I., Bellon, S., Dedieu, B., \& Milestad, R., 2010. Adaptiveness to enhance the sustainability of farming systems. A review. Agronomy for Sustainable Development, 30: 545 - 555.

Darnhofer, I; Gibbon, D., \& Dedieu, B., 2012. Farming systems research: an approach to inquiry. En: Darnhofer, Ika; Gibbon, David and Dedieu, Benoit (eds), Farming Systems Research into the 21st Century: The New Dynamic. Dordrecht: Springer, 3-31.

Folke, C., Carpenter, S.R., Walker, B., Scheffer, M., Chapin, T., \& Rockström J., 2010. Resilience thinking: integrating resilience, adaptability and transformability. Ecology and Society, 15: 20, [online] URL: http://www.ecologyandsociety.org/vol15/iss4/art20/

Holling, C.S., 1973. Resilience and stability of ecological systems. Annual Review of Ecology and Systematics 4: 1-23.

Maleksaeidi, H., \& Karami, E. 2013. Social-ecological resilience and sustainable agriculture under water scarcity. Agroecology and Sustainable Foods Systems, 37 : 262-290.

Resilience A., 2007. Assessing resilience in social-ecological systems: A workbook for scientists Version 1.1 Draft for testing and evaluation. 53 pp.

Walker, B.H., Abel, N., Anderies, J.M., \& Ryan P., 2009. Resilience, Adaptability, Transformability in the Goulburn-Broken Catchment, Australia. Ecology and Society, 14: online at http://www. ecologyandsociety.org/vol14/iss1/art12/. 